\title{
A controlled study of health education in accident prevention: the Rockland County Child Injury Project
}

Edward R Schlesinger, Donald G Dickson, Janice Westaby, Lisel Lowen, Vito M Logrillo Albert A Maiwald

The Rockland County Child Injury Prevention Project was developed as a controlled study to test the possible effects of public health education of groups of parents on the incidence of accidental injuries among their young children. Suchman and Scherzer ${ }^{1}$ have emphasized the need for controlled studies in accident prevention in childhood, especially since the future course of mortality in the age group from 1 to 15 years is largely dependent on the death rate from accidents and other violence. ${ }^{2}$ Furthermore, accidental injuries leading to death are only a small fraction of the accidents which result in temporary or permanent disability.

Three general approaches have been used in the effort to reduce the rate of accidents among children: application of engineering or environmental measures, enforcement of legal measures, and public education in safe behavior. Education may be conducted in a one-toone relationship, as is done by the practicing pediatrician with his patients, or it may be conducted through selected groups or with the community as a whole through mass media. The Rockland County project was concerned mainly with education through selected groups. Criterion of effectiveness of the education program was the rate of medically attended injuries among children under 7 years of age in families exposed to the special education program, in comparison with the rates among children of the same age in a comparable group of families exposed only to the educational influences already current in the community.

Rockland County, a rapidly growing suburban area north of New York City, offered an almost unique opportunity for a controlled study. Its numerous, relatively new housing developments peopled by young married couples with many small children provided sizable study and control groups within a convenient geographic area. A major limited access parkway dividing the county minimized the possibility of the special education program's reaching the control group. As pointed out in a preliminary report ${ }^{3}$ on the incidence of medically attended injuries during the first year of the project, the homogeneity of the population largely controlled variables such as family makeup, socioeconomic status, types of housing, and social interests.

\section{Methods}

Housing developments with 10 or more homes built not later than 1950 were divided into two similar areas of matched population, separate by a buffer zone along both sides of the limited access parkway. The selection of developments as units for the study ensured that study angु control areas would follow school district boundaries. The study group consisted of 84 developments, with 4041 homes, and the control area contained 91 developments, with 4063 homes.

A detailed survey of the data from the school census during the summer of 1962, about thes mid-point in the project, revealed a total of 4106 children under 7 years of age in the housing development in the control area an 4614 children in the developments in the study area. The proportion of children by age and sex distribution was roughly similar among the children in the study and control areas. TherE appeared to be an underenumeration of the children under 2 years of age, the under enumeration being greater among the childre壻 under 1 year of age.

Three sample surveys of homes in the studF and control areas were made during the course of the study. These were consistent in indicat? ing about a $5 \%$ turnover of families in both the study and control areas, with very few moves between the two areas.

Data on accidental injuries were obtainew directly from physicians, dentists, and hospital in the county through personal visits by dat collectors every two weeks. Treatment or care by persons other than physicians or dentists was not included in the reporting system, no was advice that was given over the telephong counted as medical or dental attention. special, simple report form was completed fop any actual or presumed trauma following an incident for which direct medical or denta? attention was obtained. A nominal fee of $\$ \overline{\mathrm{h}}$ was provided for every completed report.

The detailed methodology of the reporting procedures are outlined in the preliminarye report. $^{3}$

Following a preparatory phase of six months? data were collected on injuries for a one yea£ baseline prior to the start of the education program in December 1961. The education phase of 18 months was followed by a period of six months during which injury reporting was continued, to provide an indication of possible ongoing effects of the education program.

With the advice of an education advisory committee, a program of intesive education was developed. The basis of the program waso the organization of neighborhood discussion groups conducted by team leaders from within 
the community and by persons with professional training or specialized knowledge.

In recognition of the tendency toward apathy to 'safety' as an entity in itself, the approach to accident prevention was presented as an important component of child development and everyday living. A guide was prepared in which hazards were listed and stress was placed on preventive measures for children at different ages. Every effort was made to achieve maximum uniformity of methods, content, and exposure. A monthly newsletter was mailed to all families in the study group. Special speakers were used to a limited extent at group and club meetings outside the neighborhood discussion groups. The housing development teams consisted of two to four leaders from among the mothers, who shared the responsibility for organizing, conducting, and evaluating small neighborhood meetings. A total of 85 teams were organized in 72 developments in the study area, with 12 developments lacking team leaders.

Orientation sessions for the team leaders formed the basis for an organized program of instruction and guidance, allowing for flexibility on the part of the leaders. The neighborhood discussion meetings were held in development homes, attendance at these meetings averaging ten per meeting. Teams conducted a total of 135 meetings, 94 of these entirely on their own and 41 with specialists handling the program. The total attendance at the neighborhood meetings was 1422 , with 873 different individuals attending.

Printed materials played a significant role in the education program as an informational source for team leaders, as a guide for professional discussion leaders, and as future reference material to bolster the effects of neighborhood meetings. Selected brochures were distributed by volunteers, specially re- cruited for this purpose, in 11 of the 12 unorganized developments where no neighborhood meetings could be held.

Accident rates were computed for each three month reporting period, projected on an annual basis. The three month periods were selected to take seasonal variations into consideration, as follows: December-February, March-May, June-August, September-November.

The data collected during the four reporting periods of the baseline phase were used to determine differences, if any, in the accident rate in the study and control groups. Data collected during the six periods of the education phase were used for comparative purposes in evaluating the possible effects of accident prevention education. The final two reporting periods were related to any possible continuing effects of education. Rates were also computed separately, by reporting periods, according to source of report (hospitals or private offices of physicians and dentists), by selected types of injuries, by disposition, and by rank of severity. For the rank of severity, three groups were developed according to the nature of the injury.

In reporting the results, accident rates are defined as the rates of reported medically attended injuries per 1000 children under 7 years of age. When age- and sex-adjusted rates are given, these refer to rates among the children in the study and control groups adjusted to the population of New York State in the 1960 census.

\section{Results}

The age- and sex-adjusted accident rates for each three month period of the study revealed no consistent differences in the trends between the study and control groups during the three years of the project (fig 1). On the contrary, the

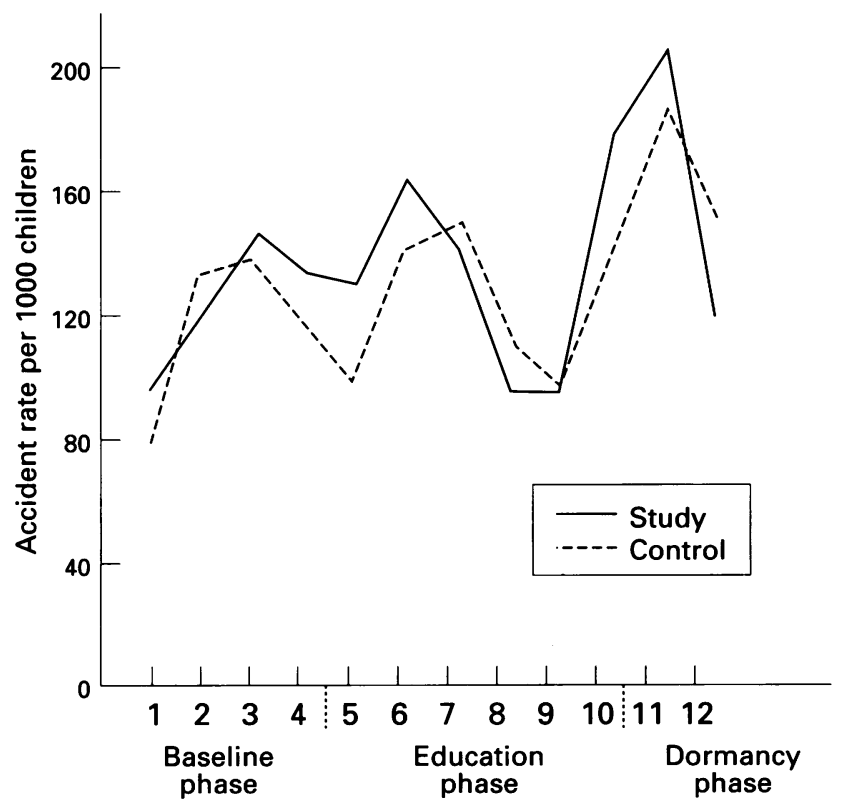

Three month reporting period

1. December 1960-February 1961

2. March 1961-May 1961

3. June 1961-August 1961

4. September 1961-November 1961

5. December 1961-February 1962

6. March 1962-May 1962

7. June 1962-August 1962

8. September 1962-November 1962

9. December 1962-February 1963

10. March 1963-May 1963

11. June 1963-August 1963

12. September 1963-November 1963

Figure 1 Total accidents by three month reporting period. Age and sex were adjusted to population of New York State, 1960 census. 
curve for this study group crossed that of the control group on no less than five occasions, with at least one of the crossovers occurring during each phase of the project.

At the end of the ninth reporting period, or 15 months after the start of the education program, the trend in the differences in the accident rates in the study and control groups suggested the possibility of some impact on the incidence of accidents in the study group following exposure of their parents to the education program. If this difference had continued as a consistent trend, it would clearly have suggested a positive relationship betwen the education program and the rate of accidents among the young children whose parents had been exposed to such a program. Any conclusion relating to the positive effect of the education program proved untenable, however, in the light of the sharp and disproportionate increase in the accident rate in the study group during the tenth and 11th reporting periods. The developments during these two periods, after what appeared to be an earlier contrary trend, points vividly to the need for continued reporting over a sufficient time, in a controlled situation, to be certain that fortuitous changes over short periods do not lead to unwarranted conclusions.

The sharp swings in the accident rates among both study and control groups over the three year period of the study are apparently related mainly to seasonal factors, with the high points in the curves occurring in the spring and summer months. With the exception of the dip in the ninth quarter, there appeared to be a moderate uptrend of similar proportions in the recorded accident rates in both the study and control groups. The upward trends may well have been artificial and due, at least in part, to the underenumeration of children under 2 years of age from the population data obtained from the school survey. Since the population distribution for the final year of the project was estimated by moving the school survey population ahead by a year, resulting smaller numbers of children at ages of higher accident incidence would tend to increase the total rate in both the study and control groups.

While the overall rate of accidents showed no apparent changes in the study versus control groups during or following the education program, significant changes might actually have occurred which might not have been reflected in the overall accident rates. It was possible, for example, that the rate of more serious accidents might have decreased in the study group, but that this decrease might have been obscured by a corresponding or greater increase in the number of visits for medical attention for trivial injuries resulting from increased interest and awareness on the part of the parents after their exposure to the education program. The possibility that changes might have occurred in certain types of accidents or in specific age or sex groups also required investigation, if only to eliminate these factors as having any bearing on the total accident rates.

The most significant of these factors ap- peared to be the severity of the injury for which attention was sought. This was first approached through analysis of the proportion of accidents in which a return visit was recommended by the physician or dentist of in which hospitalization occurred. The trends. in the proportion of these presumably more severe injuries are presented, by reporting period, in fig 2 . No change in either the studg or the control group is apparent from these curves. The curve for the study group was essentially flat for the entire period of the project. While there was considerably morê variation by reporting periods in the percentage $\vec{D}$ in the control group with recommended return visits or hospitalization, the overall trend dify not change. Separate analysis of the proportiox of children hospitalized again revealed no clearu trend in either the study or control group; the proportion hospitalized in the study grour exceeded that in the control group in six of the eight reporting periods during the education and dormancy phases of the project.

Ranking the severity of the injury for whic attention was sought was another approach to the possible detection of finer changes in the rates. Multiple injuries were coded by the most severe injury reported. The division of severity of type of injury into mild, moderate, and severe, while broad and somewhat arbitrary. provided a basis for comparison between the study and control groups. In fig 3, the graphio presentation of the trends in the proportion of injuries by degrees of severity, no discernible change is evident in either the study or contro population or in the difference between these two population groups in any of the thre severity groups.

No significant changes could be detected in the trends of the rates for particular types of accidents in the study versus the controథ groups. This applied equally to falls, which may be the least amenable to prevention through education, and to burns and scald and poisoning, which might be most influo enced by a preventive approach througl education.

To achieve the fullest reporting of medicalle attended injuries, every possible professiona $\Phi$ source of reports was considered in planning the project. At the time the project was planned, reports from the emergency rooms

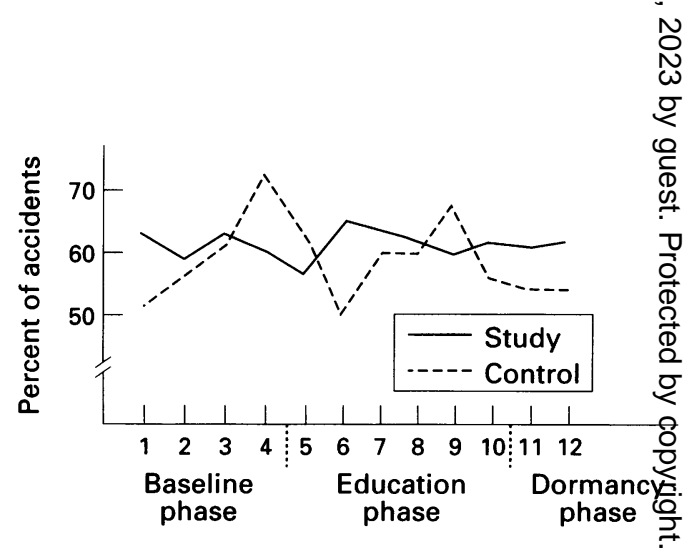

Figure 2 Accidents involving more than one visit or hospitalization by three month reporting period. 


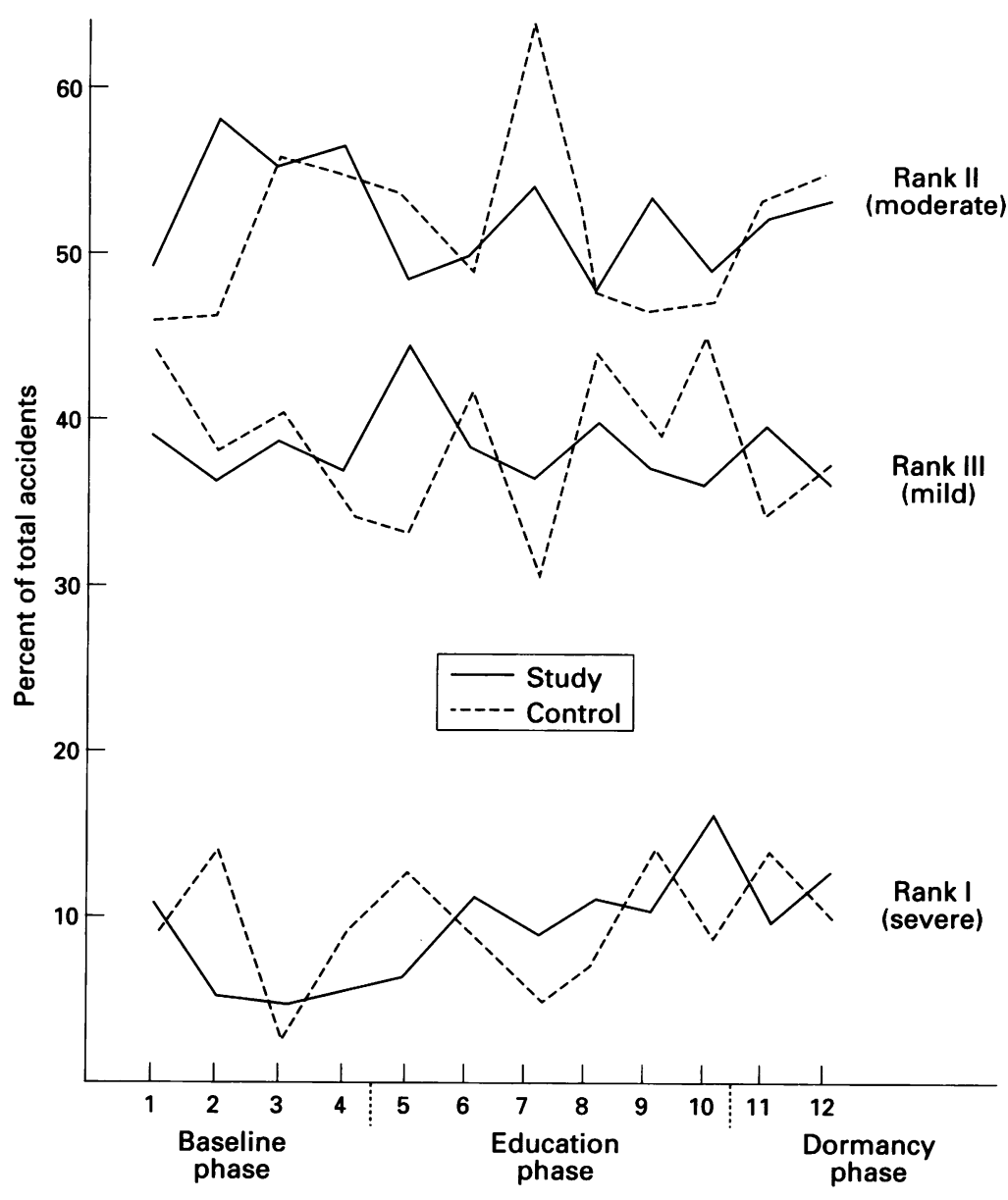

Figure 3 Accidents by severity of nature of injury by three month reporting period.

of hospitals were not thought to be adequate as the sole source of reporting. Hence, reports were to be obtained from practicing physicians and dentists as well as from hospitals.

The reports from hospitals may be accepted as approaching completeness, since the data collectors actually went over the emergency room records completely with the responsible person in each hospital. The trends in the accident rates in the study and control groups as obtained from hospital reports are shown in fig 4. The closeness in the correspondence of the curves for the accident rates for the study and control groups is striking, and the seasonal pattern of accident incidence stands out sharply.

On the other hand, the curves for accident rates based on reports from private physicians (fig 5) deviate much more widely from each other, and seasonal fluctuations are scarcely evident at all. These finding suggest that the flow of reports from physicians is much more erratic than from hospitals. Even though the reports were obtained from physicians through periodic visits to their offices, and a small fee was provided for each, completion of the reports still required a special effort on the part of a physician or an office assistant. Unless the constant interest of the physician was maintained, there was a good chance that the records of varying numbers of children with injuries would be overlooked. It is also possible that the regular, sharp peaks in the reports obtained from the hospitals during the summer months in the third, seventh, and 11 th reporting periods may have been a reflection of the relative convenience in obtaining emergency care in the hospital during the vacation season.

The results suggest that, in a controlled study of the nature of this project in the particular area selected, adequate rates for comparative purposes might have been obtained from reports gathered solely from hospital records. Increasing the size of the study and control groups would have compensated for the smaller number of accidents involved. Theoretically, this could have been done without necessarily increasing the cost of the project, since some of the funds needed for the intensive efforts in data collection would then have been available for expanding the health education program to a larger study group. In actuality, however, this would not have been possible, since all the housing developments suitable for study were already included in the project.

\section{Comments}

The one clear conclusion that may be drawn from this study is that the incidence of medically attended injuries from accidents was not reduced during or after an education program directed specifically at parents of young children in a select population group, and that this absence of any demonstrable impact of the program was evident in the study group itself and in comparison with a control group of generally similar character. While general conclusions of the effectiveness of health education in changing human behavior toward safer practices of daily living should not be drawn from this one study, it does point up the difficulties involved. The study demonstrates graphically the importance of controls in any such study involving the behavior of human population groups, and it further reinforces the need to have such studies continued for a sufficiently long period of time

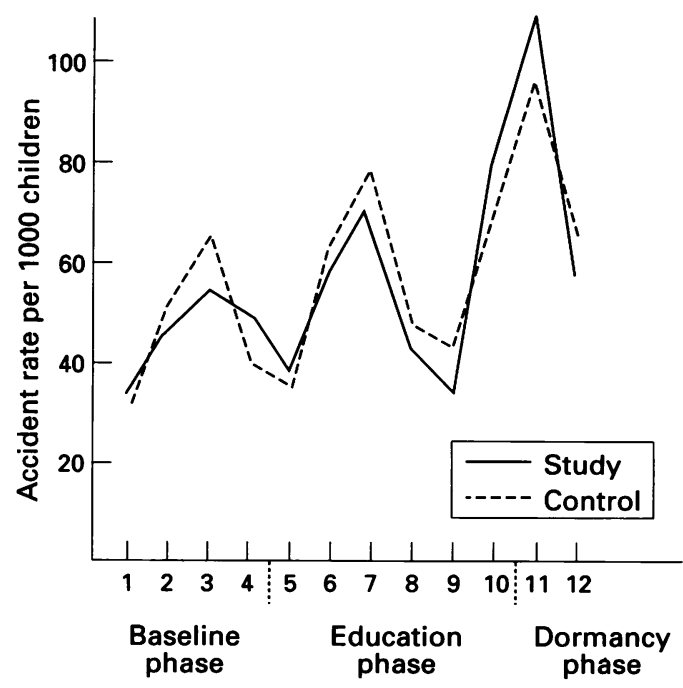

Figure 4 Accidents from all hospital reports by three month reporting period. 


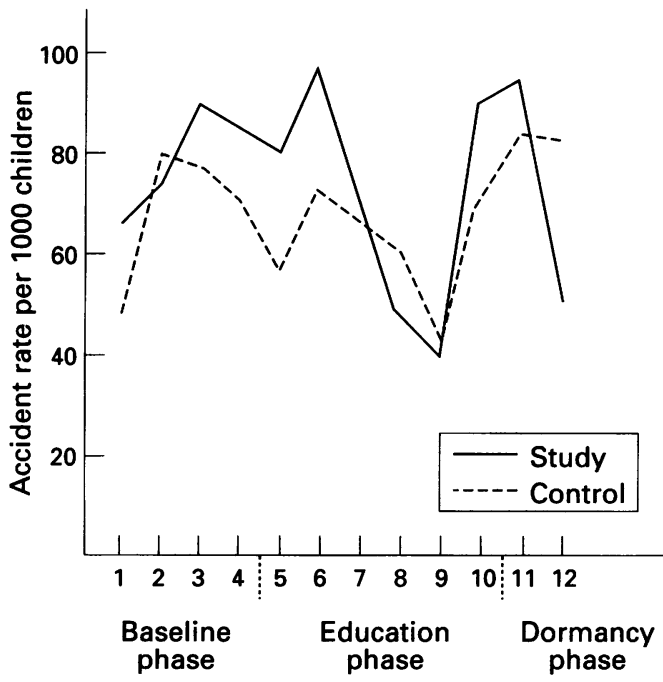

Figure 5 Accidents from reports from physicians and dentists by three month reporting period.

to obviate the temptation to draw premature conclusions regarding favorable results.

Epidemiological questions in this study deserve careful consideration in the design of similar studies. The selection of medical or dental attendance for an accidental injury as the criterion of a measurable accident necessarily results in inclusion of injuries of a trivial nature. Furthermore, parents vary widely in their determination of the severity of an injury for which they seek medical attention for their children. However, other possible definitions of accidents have equal or possibly greater limitations. Selection of deaths from accidental causes as a criterion would have necessitated an impossibly large study and control population. Furthermore, the selection of death as a criterion of the success or failure of educational efforts may itself be open to serious question. Whether or not death occurs as the end point of an accident is related to many other factors superimposed on the occurrence of the original accident. The adequacy of first aid measures at the site of the accident, the precautions observed in transporting the injured person for definitive care, and the speed with which definitive medical attention is obtained are phases of secondary prevention which may play as important a role in determining the outcome as prevention of the accident itself.

A philosophical question relates to the desirability of intensive efforts to prevent minor injuries which might be considered almost a necessary part of the learning process in the child's development. Cullen has suggested that there may even be a positive gain from minor accidents. 'For the toddler with little understanding of how and why it happened, it can be the occasion for the reaffirmation of his parents' love and concern for him. For the older child confronted by a distinctly unpleasant side of reality - pain - there is a chance to learn to tolerate something which hurts, incapacitates, and thwarts'.

On the other hand, there are many minor injuries which, for a seemingly trivial difference in the circumstances, might have led to permanent disability or death. Most of the falls in the study, particularly those occurring on the same level, resulted in very minor contusion $\overline{\mathrm{s}}$. or abrasions, but they could just as readily have led to fractures, facial injury, or punture wounds.

The objection might be raised that the particular educational techniques employe $\bar{\Phi}$ were not intensive enough to expect that the would have any effect on behavior to the extent of reducing accidents. However, the fact that 135 neighborhood meetings were held, iø which there was a high degree of activg participation, indicates an intensity of effort which can rarely be attained under conditionsy of ongoing community accident prevention programs. In essence, the project was dealing with an aggregate of small communities with ga population of about 15000 (the total popula tion in the households included in the stud $\$$ group). If this number of meetings of similar size were to be held in a town of $1500 \vec{\theta}$ population this would be accepted as at intensive public health education effort. Or ientation of 250 team leaders and helpers, most of whom were active in the project, was itself $\stackrel{\mathbb{a}}{a}$ significant educational activity.

The Rockland County project dealt with the education of groups of parents of young children, and therefore should not be consid? ered as equivalent to efforts with individuaf parents. It is possible that a one-to-one relationship between a physician or some othe authoritative person and the mother might bet more effective in preventing accidents in young children. A recent controlled study by Bass and his associates ${ }^{5}$ suggests that advice on the installation of seat belts in cars, at the time of regular visits for pediatric health supervision, is followed by the desired action nearly three times more often than in control groups wh\$ are only sent letters on the same subject of given no advice at all by the physician However, the study group was not necessarily comparable to the control group, since the former may have been more highly motivate in that they had come to the physician's offices during the period of the study. Furthermore the desired action was the installation of the seat belts, a one time act, and not a complex and prolonged change toward safer behavior In point of fact, no attempt was made tब discover whether the seat belts were even used consistently after installation.

The Rockland County project did now attempt to provide a universal answer to the effect of education on the incidence of accic dents. It could test only the effect of a broa糔 educational approach on the incidence of accidents among young children in a specifie population group - children of young parents living in relatively new suburban develop@ ments. Indeed, the self selection of the parent? for the educational program may have resulted in a group with a particularly high level o⿺廴⿻肀二 interest and knowledge regarding safe beha vior, so that any further reduction in acciden? incidence among their children could, at best, be minimal. 


\section{Summary}

The Rockland County Child Injury Prevention Project was designed to test the effects of public health education for parents on the incidence of accidental injuries to children under 7 years of age in a controlled situation. The incidence of accidental injuries, defined as any actual or presumed trauma following an accident for which medical or dental attention was obtained, was determined in a study population before, during, and after exposure to the educational program and in a comparable control group during a corresponding period of time.

The study group was exposed to an intensive education program involving neighborhood discussion groups conducted by lay and professional leaders, meetings with organized groups, and a monthly newsletter. The study population was organized into small neighborhood units of about 24 homes within the suburban housing developments.

To measure the effect of the education program, accident rates were computed for each three month reporting period, projected on an annual basis. No consistent differences were discernible in the trends in the accident rates betwen the study and control groups during the three years of the project. On the contrary, the curve for the study group crossed that of the control group on no less than five occasions. There was a sharp decline in accidents during the second half of the education phase.

Any conclusions regarding a positive effect of the education program proved untenable in the light of the sharp and disproportionate increase in the accident rate in the study group during the six month phase after education had stopped. After what appeared to be a contrary trend earlier, developments during this period demonstrated vividly the need to continue reporting for a sufficient time in a controlled situation to be sure that fortuitous changes over short periods do not lead to unwarrented conclusion.

The flow of reports from hospitals was more consistent than from physicians. This suggested that, in selected control studies, adequate rates for comparative purposes might have been obtained entirely from hospital records.

This study was supported by grant 00056-04, Division of Accident Prevention, US Public Health Service, for the period September 1959 through April 1964.

For copies of the complete project report, write to Rockland County Department of Health, County Office Building, New City, NY 10956 (Dr Dickson).

1 Suchman E, Scherzer AL. Current research in childhood accidents. New York: Association for the Aid of Crippled Children, 1960

2 Moriyama IM. The change in mortaility trend in the United States: vital and health statistics analytical studies. Series 3 , No 1. Washington: National Center for Health Statistics, Public Health Service, March 1964.

3 Dickson DG, Schlesinger ER, Westaby JR, Lowen L. Medically attended injuries among young children: 107: $618-23$.

4 Cullen JS. The evaluation of accidents to childdren. $A m \mathcal{F}$ Public Health 1961; 51: 990-7.

5 Bass LW, Wilson TR. The pediatrician's influence in private practice measured by a controlled seat belt study. Pediatrics 1964; 33: $700-4$. 